\title{
Politique
}

\section{Sommaire des thèses 1986-1987}

Numéro 12, automne 1987

Mouvements et acteurs

URI : https://id.erudit.org/iderudit/040581ar

DOI : https://doi.org/10.7202/040581ar

Aller au sommaire du numéro

Éditeur(s)

Société québécoise de science politique

ISSN

0711-608X (imprimé)

1918-6584 (numérique)

Découvrir la revue

Citer ce document

(1987). Sommaire des thèses 1986-1987. Politique, (12), 169-174.

https://doi.org/10.7202/040581ar

Ce document est protégé par la loi sur le droit d'auteur. L'utilisation des services d'Érudit (y compris la reproduction) est assujettie à sa politique d'utilisation que vous pouvez consulter en ligne.

https://apropos.erudit.org/fr/usagers/politique-dutilisation/
Cet article est diffusé et préservé par Érudit.

Érudit est un consortium interuniversitaire sans but lucratif composé de l’Université de Montréal, l'Université Laval et l'Université du Québec à Montréal. Il a pour mission la promotion et la valorisation de la recherche. https://www.erudit.org/fr/ 


\section{Sommaire des thèses 1986-1987}

\section{Université Laval}

Beauchamp, Michel, «Communication, pouvoir et organisations coopératives: le cas du Mouvement Desjardins».

Dans le but de mieux évaluer l'importance de certaines activités de communication à caractère stratégique dans le développement du Mouvement des caisses Desjardins, depuis 1900, trois propositions de recherche ont été appréciées lors de l'étude de trois cas: les caisses scolaires, une activité de propagation; les campagnes publicitaires de 197778 et 1978-79; l'offre du Régime d'épargne-retraite, une activité de promotion.

Il est globalement ressorti de l'analyse des trois propositions que ces activités de communication, qui ont représenté une source de pouvoir et un enjeu importants pour le Mouvement Desjardins, ont influé sur la dynamique interne de cette organisation, marqué les relations établies avec divers segments de l'environnement et constitué un révélateur privilégié de ces mêmes relations.

Diop, Abdoulaye, «Environnement et changement politique: le multipartisme sénégalais".

Il s'agit principalement, dans cette recherche, d'analyser la dynamique politique sénégalaise pour comprendre et expliquer l'instauration du multipartisme au Sénégal. Notre objectif a été de montrer que le multipartisme sénégalais est une réponse à une exigence d'équilibration, à une exigence d'adaptation et à une exigence de légitimation. Nous avons pris en considération la période 1960-1983 pour mieux établir les enchaînements et les ruptures entre les différents éléments et avons utilisé l'approche systématique pour l'analyse des données. Les documents écrits ou chiffrés traitant directement ou indirectement du sujet ont été consultés. Les témoignages ont été pris en considération. Nous avons fait appel à l'analyse documentaire principalement aux méthodes classiques et aux méthodes quantitatives pour utiliser cette documentation. Nous avons étudié, dans cette recherche. l'historique du système partisan sénégalais (du multipartisme au monopartisme), la politique de centralisation du pouvoir et ses conséquences, les conditions d'existence de la démocratie, du multipartisme et les thèses sur l'origine des partis retenues par les auteurs. Nous avons aussi étudié la théorie générale du système et l'approche systémique en politique. L'analyse des données s'est faite principalement à l'aide de l'approche systémique et s'est articulée autour de l'étude des inputs, des outputs et de la rétroaction. Au total, notre recherche montre que le multipartisme sénégalais constitue une réponse à une exigence d'équilibration, à une exigence d'adaptation, à une exigence de légitimation et est le résultat de la capacité d'auto-organisation du système politique sénégalais. 
Traore, Oumar Aba, «Le parti unique et l'objectif de construction de l'unité nationale: le cas de l'U.D.P.M. au Mali».

La construction de l'unité nationale a souvent été présentée comme l'objectif fondamental que vise le parti unique eu égard à l'hétérogénéité des sociétés africaines. Considérant cette allégation générale de fortuite et sans fondement véritable, nous avons décidé - à l'aide des techniques d'analyse et des indices d'évaluation de l'unité nationale - d'entreprendre un travail de recherche en vue d'aboutir à des résultats convaincants. Dans cette optique, nous avons choisi de faire une étude de cas basée sur l'U.D.P.M. au Mali qui a révélé, après analyse exhaustive, que le Mali n'est pas une "nation" au sens moderne du terme, contrairement à ce que pensent les dirigeants du parti unique malien; qu ele parti de l'U.D.P.M. ne vise donc pas globalement et d'une façon cohérente à construire l'unité nationale. L'étude a également montré que le parti unique a été institué non pas par souci de satisfaire des objectifs d'unité nationale mais bien par respect des traditions et par peur également de provoquer au sein de la population du Mali des querelles fratricides.

\section{Université de Montréal}

Tremblay, Pierre P. «Loteries et attitudes politiques au Québec».

En décembre 1969, le gouvernement du Québec se dotait d'une société d'État chargée de l'exploitation des jeux de loterie. Depuis ce temps, la popularité de ce jeu n'a pas cessé de crô̂tre. L'engouement des Québécois envers la loterie a suscité plusieurs commentaires et analyses, tantôt favorables et tantôt défavorables. Qui a raison? Qui a tort? À vrai dire, nous n'en savons rien.

Des chercheurs, à la suite d'E.C. Devereux, ont développé et articulé un concept propre aux jeux de hasard et d'argent: le concept de soupape de sécurité. Un autre auteur, la suédoise Nechama Tec, a vérifié l'incidence de l'habitude du jeu sur la qualité et l'intensité du comportement politique des individus, sa conclusion: il n'y a pas de lien véritable entre les deux objets. Puis, Gabrielle et Reuven Brenner, deux économistes québécois, ont élaboré un modèle destiné à expliquer les incitatifs à la consommation de loterie. Ce modèle prédit que ce sont les gens les plus pauvres qui achètent davantage de billets de loterie.

Nous avons voulu mettre a l'épreuve le bien-fondé de ces diverses théories tout en mesurant l'impact de la consommation sur les attitudes politiques. Reprenant la thèse de la soupape de sécurité, nous avons élaboré l'hypothèse suivante: si l'achat régulier de billets de loterie est une manifestation de l'insatisfaction de la condition socio-économique ainsi que du désir de posséder les valeurs socio-économique ainsi que du désir de posséder les valeurs importantes du système générateur de cette insatisfaction, l'habitude régulière de la loterie constitue une manifestation de l'adhésion au système et à ses valeurs. Donc, la loterie est, en ce sens, une soupape de sécurité et renforce les attitudes conservatrices des individus.

De l'hypothèse centrale, il a été formulé cinq prédictions, à savoir:

1. Tout affaiblissement de la conjoncture économique entraîne l'augmentation de la consommation de loterie; 
2. Les individus les plus pauvres économiquement achètent proportionnellement plus de billets de loterie;

3. L'incitation principale à la consommation de loterie est le désir de gagner rapidement de l'argent et de devenir riche;

4. Les acheteurs de loterie accordent plus que les autres une grande valeur à l'argent;

5. L'habitude régulière de la loterie entraîne un taux plus élevé d'attitudes politiques conservatrices.

Nous avons testé cet ensemble théorique par voie de recherches documentaires, d'enquêtes par sondage et par analyses statistiques.

Telle que libellée, l'hypothèse de recherche a dû être rejetée. Bien qu'il se soit révélé évident que l'achat de loterie est la manifestation du désir de devenir riche, il n'est pas, en retour, l'expression de l'insatisfaction de la condition économique. De plus, si nous avons constaté une adhésion certaine envers le système et la société, nous n'avons pas, en contrepartie, observé un lien entre la consommation de loterie et le conservatisme. Les résultats de nos travaux nous ont amenés à conclure que la loterie constitue une soupape de sécurité mais diffuse et essentiellement de caractère économique. En cela, la loterie exerce une fonction différente de celle prédite par Devereux et Zola.

Le rejet des première et seconde prédictions, découlant du modèle économique de Brenner et Brenner, nous a forcé à invalider ce modèle.

La troisième prédiction axée sur le motif du désir de l'argent s'est avérée être la seule confirmée. Ce résultat laisse entrevoir la loterie comme un produit surtout économique et en rien ludique.

Le rejet de la quatrième prédiction nous a amené à constater une absence de démarcation entre les joueurs et les non-joueurs au chapitre des valeurs. Ce résultat donne du poids à l'argument qui voit la consommation comme adhésion au système de valeurs.

Enfin, le rejet de la dernière prédiction est celui qui nous oblige à invalider l'hypothèse centrale. En retour, ce résultat nous permet d'avancer le caractère économique de la loterie en tant que soupape de sécurité.

En conclusion, les résultats de notre recherche, en plus de préciser le caractère surtout économique de la thèse de la soupape de sécurité, invalident le modèle de Brenner et Brenner et prolongent dans le champ des attitudes politiques les constatations de Nechama Tec sur l'absence de relation entre le jeu et le comportement politique.

Bouchard, Gilles «Les facteurs d'influence dans les relations entre fonctionnaires et citoyens».

Le point de départ de cette thèse était la recherche des facteurs qui influencent les relations fonctionnaires-citoyens. Pour ce faire, un cadre général d'analyse a été élaboré. Dans ce cadre, la relation fonctionnaires-citoyens a été isolée et considérée comme la variable dépendante. Celle-ci est soumise à l'influence de facteurs externes qui se situent soit dans l'organisation impliquée dans la relation, soit dans l'environnement immédiat de cette organisation. Ces facteurs constituent les variables indépendantes de cette recherche. L'influence de chacun de ces facteurs n'est pas considérée isolément. Au contraire, les facteurs seront réunis pour former des ensembles divers appelés modèles et c'est l'influence de ces ensembles sur la relation qui sera étudiée. Le choix des facteurs devant former un modèle sera fait en tenant compte de la convergence de leurs effets sur la relation. 
L'hypothèse centrale de la thèse s'énonce donc comme suit: un ensemble de facteurs environnementaux et organisationnels dont les effets sur la relation sont convergents détermine les caractéristiques de la relation qui s'instaurera entre les fonctionnaires et les citoyens.

Une revue de la littérature sur les relations entre l'organisation et son environnement a permis d'identifier les facteurs les plus souvent retenus comme influençant la relation. De ces facteurs, neuf variables ont été précisées et pour chacune d'elles des indicateurs ont été constitués. La variable linguistique entend prendre en compte le degré d'homogénéité linguistique entre fonctionnaires et clients. La variable classes sociales identifie la classe sociale auquelle appartient la clientèle. La variable concurrence examine dans quelle mesure l'organisation étudiée est en position de monopole face à sa clientèle. La variable groupement s'intéresse au degré d'organisation de la clientèle. La variable dispersion entend évaluer dans quelle mesure la clientèle est regroupée sur le territoire. La variable contact considère le caractère volontaire ou obligatoire de la relation. La variable spécialisation s'intéresse au degré de professionalisation des fonctionnaires alors que la variable déconcentration essaie d'évaluer la distance entre fonctionnaires et clients. Enfin la variable taille tient compte de la grosseur de l'organisation.

Quant à la relation, elle comprend trois éléments soit les relais, les services spécialisés et la communication. Les relais sont les groupes ou individus qui sont reconnus par l'organisation comme interlocuteurs ou porte-parole de la clientèle. Deux aspects de ce premier élément seront examinés soit la quantité de relais et leur degré de représentativité par rapport à l'ensemble de la clientèle de l'organisation. Les services spécialisés sont les services qui, dans l'organisation, remplissent le rôle de relations publiques. Deux types de services seront retenus et évalués soit les conseils consultatifs et les services d'information. Dernier élément, la communication correspond en gros en ce qu'en théorie des communications on appelle les messages et les canaux. Trois aspects de cette communication seront considérés soit son caractère formel ou informel, sa direction et le degré de satisfaction émise à son endroit par les acteurs.

À partir des effets prévisibles de chacune des neuf variables sur les divers éléments de la relation, des modèles ont été élaborés, cinq au total. Ces modèles ont reçu comme appellation une lettre de l'alphabet. Le modèle $A$ devait regrouper les organisations s'adressant à une clientèle bourgeoise, le modèle $\mathrm{Z}$ à une clientèle défavorisée, le modèle $P$ à une clientèle petite bourgeoise et le modèle I à une clientèle institutionnelle. Le modèle $\mathrm{B}$ regroupait quant à lui les variables favorisant une relation bureaucratique.

Avec ces modèles comme guide, tous les ministères des gouvernements du Québec et du Nouveau-Brunswick ont été passés en revue. Dix de ceux-ci ont été retenus car ils se rapprochaient des modèles. Un certain nombre d'hypothèses ont été émises sur les relations que ces ministères seraient susceptibles d'entretenir avec leurs clientèles. Après vérification, soixante-quinze pour cent des hypothèses se sont révélées exactes.

En conclusion, bien que l'exercice piusse apparaître criticable en particulier en ce qui a trait aux choix des variables significatives et de leurs indicateurs, les résultats obtenus sont intéressants à bien des égards. Premièrement, le haut pourcentage de vérification des hypothèses est certes une indication de l'utilité de considérer les effets convergents de plusieurs variables. Deuxièmement l'utilisation de modèles a permis de faire ressortir l'existence au sein des gouvernements provinciaux de types de ministères différents tant au niveau de leurs caractéristiques internes et de celles de leurs clientèles qu'au niveau de leurs relations. Enfin cette étude démontre qu'il est possible d'aller chercher une série 
de données intéressantes mais elle en indique aussi les limites et nous amène à nous interroger si de tels exercices ne nécessitent pas un travail d'équipe plutôt qu'une recherche individuelle.

\section{Université McGill}

Gidengil, Elizabeth «Class and region in Canadian voting behaviour: a dependency interpretation".

"A theoretical rationale for the interplay between class and region in influencing Canadian voting behaviour is developed in terms of a dependency interpretation of the phenomenon of regional disparity in Canada. Dependency is conceptualized as a syndrome of related characteristics, its components identified as external penetration, restricted choice, vertical interaction, and structural distortions.

Cluster analysis is used to develop an objective classification of sub-provincial regions in Canada according to the similarity in their profiles on seven operational measures of the dependency syndrome. This classification forms the basis for an examination of the interaction between class and region in Canadian voting, using data from the 1965, 1968 , and 1974 national election studies.

Additional support is offered for the validity of a dependency interpretation of regional effects by demonstrating the existence in Canada's disadvantaged regions of a political culture of dependency."

Laforest, Guy Les sciences humaines modernes: diversité épistémologique et complémentarité politique.

This work wishes to call into question two propositions about the modern human sciences: the monopolistic pretentions alternately formulated by explanation and interpretation, thereafter the attribution of exclusively rational foundations of these knowledges. It examines first the history of explanatory and interpretive disciplines, emphasizing the unmistakable epistomological diversity which characterizes this scientific field. The questions of the foundations of knowledge, of the nature of truth, are then explored; this leads to the reformulation of Foucault's argument, which affirms that the universe of discourse would not be sufficient to give an account of the foundations of the human sciences, neglecting their close intrication with social practices and relations of power. Guided by Foucault's last writings, the thesis tries to find the complementarity between the explanatory and interpretive human sciences in their participation in a world-wide movement of normalization.

Goldberg, David «Ethnic Interest Groups as Domestic Sources of Foreign Policy: A Theoretical and Empirical Inquiry».

This study investigates the phenomenon of ethnic interest groups as domestic sources of influence on the making of foreign policy on a cross-national basis. The attempt is made first to develop a framework for comparing theoretically the role of ethnic groups in various governmental systems. Once completed, the various conceptual assumptions 
are applied to the activities of domestic ethnic interest groups in the United States and Canada concerned with policy for the Middle East and the Arab-Israel conflict. The focus is primarily on the American and Canadian pro-Israel lobbies during the period between October 1973 and September 1982. Data for domestic Arab ethnic constituencies are also considered where relevant, but more as logical counter-points-to the North American Jewish communities than as bases for full and complete cross-ethnic comparison. The principal objective of this study is to compare the political influence of two interest groups of the same faith and fundamental purpose but of different systems of government and political cultures.

Sae-Jung, Kim «The Political Economy of Authoritarianism: State-Propelled Industrialization and the Persistent Authoritarian State in South Korea, 1961-1979».

En tenant compte de la spécificité du développement parallèle de la modernisation industrielle et de l'intensification de l'autoritarisme politique en Corée du Sud sous la direction de Park Chung Hee - ce qui remet en question la conceptualisation du changement politique de la théorie conventionnelle, qu'elle soit libérale ou marxiste cette thèse propose son propre modèle explicatif qui intègre les hypothèses étatiques et "late-comer» de façon dynamique. Conformément à ce nouveau modèle, cette thèse tente de repérer la configuration particulière d'interaction des sphères politique, économique et sociale en Corée du Sud entre 1961 et 1979.

La thèse soutient dans ses conclusions générales que les exigences politiques de l'industrialisation tardive, qui fut encouragée par l'État dans un contexte historique particulier caractérisée par l'absence d'une bourgeoisie dynamique, ainsi que les effets ambigus de l'industrialisation, constituent des facteurs d'élucidation importants de la spécificité du cas coréen.

En arrivant à cette conclusion, la thèse aborde quatre domaines d'importance théorique: (1) la relation fonctionnelle entre le pouvoir étatique autonome centralisé et l'industrialisation tardive; (2) la nature de l'État; (3) les implications des effets ambigus de l'industrialisation dirigée par l'État et (4) la nature et les causes des réagencements du pouvoir dans l'État autoritaire. 